Revista Brasileira de Agricultura Irrigada v.4, n.4, p.186-196, 2010

ISSN 1982-7679 (On-line)

Fortaleza, CE, INOVAGRI - http://www.inovagri.org.br

Protocolo 013.120

\title{
CRESCIMENTO DO SISTEMA RADICULAR DE CANA-DE-AÇÚCAR SUBMETIDA A LÂMINAS DE IRRIGAÇÃO: MODELO GEOESPACIAL E INFLUENCIAS NA PRODUTIVIDADE DA CULTURA
}

\author{
C. H. de A. Farias ${ }^{1}$, K. S. de Sousa ${ }^{2}$, I. de F. da Silva ${ }^{3}$, R. V. Agra ${ }^{4}$, G. da C. G. Neto ${ }^{5}$ \\ ${ }^{1}$ Eng $^{\circ}$ Agr $^{\circ}$ Dr. Pesquisador da Destilaria Miriri S.A. e-mail: carlos.henrique@miriri.com.br \\ ${ }^{2}$ Eng $^{\circ}$ Agr $^{\circ}$ Mestrando em manjo de solo e água da UFPB \\ ${ }^{3}$ Prof. Dr. Departamento de Solos e Engenharia Rural CCA/UFPB \\ ${ }^{4}$ Aluno do curso de Engenharia de Minas \\ ${ }^{5}$ Eng $^{\circ}$ Agr $^{\circ}$ Doutorando em Engenharia Agrícola UFCG
}

RESUMO: Com o objetivo de avaliar o crescimento do sistema radicular da cana-de-açúcar quando submetida a níveis de irrigação (sequeiro, 50, 75, 100 e 125\% da ETc), instalou-se uma pesquisa em área experimental (latitude 6 ${ }^{\circ} 54$ '59,88" S, longitude 3509'17,86" O e altitude de 121,00 m) da Fazenda Capim II, localizada no Município de Capim/PB, Foram coletadas amostras no plano tridimensional $(\mathrm{x}, \mathrm{y}, \mathrm{z})$ com três repetições em cada tratamento de irrigação; cada uma delas com um total de 216 amostras de solo com raízes. Para a amostragem foi utilizada um trado holandês para extração de raízes com dimensões de 7,62 x $15,00 \mathrm{~cm}$. As amostras foram coletadas em um cubo de $1 \mathrm{~m}^{3}$ onde, ao centro, situou-se a linha de cana. O espaçamento entre amostras no plano $\mathbf{x y}, \mathbf{x z}$ e yz foi de $20,00 \mathrm{~cm}$ e no plano zc foi de $15,00 \mathrm{~cm}$ (profundidade). Procedeu-se com as análises estatísticas e de geoestatística. O crescimento radicular da variedade RB 92579 não é aleatório e tem dependência espacial de moderada a forte com alcance entre 23,00 a 28,00 cm de acordo com o tratamento. A irrigação promoveu superficialização da zona de alta densidade radicular e favoreceu o aumento de raízes em zonas de baixa densidade.

Palavras-chave: raízes, geoestatística, dependência espacial

\section{GROWTH OF THE ROOT SYSTEM SUGARCANE UNDER DEPHT IRRIGATION: A GEOESPTIAL MODEL I}

\begin{abstract}
: to evaluate the root growth of sugarcane when subjected to water depths (Rainfed, 25 and 50\% ETc), installed an experimental research area (latitude $6^{\circ} 54 ' 59,88$ "S , longitude $35^{\circ} 09^{\prime} 17,86$ "and the elevation of $121.00 \mathrm{~m}$ ) Capim II Farm, located in the City of Capim/PB, samples were collected in the 3D plane $(\mathrm{x}, \mathrm{y}, \mathrm{z}, \mathrm{c})$ three repetitions of each irrigation treatment, each with a total of 216 soil samples with roots. To sample an auger was used for extraction of dutch roots with dimensions of $7.62 \times 15.00 \mathrm{~cm}$. The samples were collected on a cube of $1 \mathrm{~m}^{3}$ where the center stood a row of cane. The sample spacing in the xy plane, $\mathrm{xz}$ and yz was $20.00 \mathrm{~cm}$ and the zc plane was $15.00 \mathrm{~cm}$ (depth). Proceeded with the usual statistical analysis and geostatistics. Root growth of variety RB 92579 is not random and has a spatial dependence moderate to strong with a range between 23.00 to 28.00
\end{abstract}




\section{CRESCIMENTO DO SISTEMA RADICULAR DE CANA-DE-AÇÚCAR SUBMETIDA A LÂMINAS DE IRRIGAÇÃO: MODELO GEOESPACIAL E INFLUÊNCIAS NA PRODUTIVIDADE DA CULTURA}

$\mathrm{cm}$ according to the treatment. Irrigated superficial zone of high root density and favored the growth of roots in zones of low density.

Keywords: roots, geostatistics, spatial dependence

\section{INTRODUÇÃO}

A cultura da cana-de-açúcar (Saccharum spp.) é vista pela comunidade científica como a mais viável saída, em termos de biocombustível, que tem a sociedade para reduzir gradativamente as emissões de $\mathrm{CO}_{2}$ advindas do sistema de transporte global. $\mathrm{O}$ Brasil assume liderança em termos de produção ocupando a primeira posição. $\mathrm{O}$ Nordeste do país é responsável por produzir $11,26 \%$ da produção nacional de cana-deaçúcar, sendo a Paraíba responsável por $1,03 \%$ da mesma (ÚNICA, 2010).

No Estado paraibano a região produtora de cana-de-açúcar fica centrada no Tabuleiro Costeiro. Estudos de Farias et. al (2004) e Farias \& Oliveira (2007) relatam a fragilidade dos solos dessa região; problemas com elevada saturação de alumínio no solo, baixa fertilidade e déficit hídrico, são problemas para a produção canavieira dessa região; que terminam por afetar o desenvolvimento da planta, sobremaneira crescimento radicular da canade-açúcar.

Morris e Tai (2004) concluíram que os melhores rendimentos estão sempre relacionados à menor concentração de raízes curtas e de diâmetro maior na superfície e maior concentração de raízes longas e mais finas nos horizontes mais profundos do solo. Para Otto et al. (2009), 65,00\% das raízes de cana encontram-se nos primeiros $20,00 \mathrm{~cm}$ de solo. Já Farias et al. (2008) verificaram, com a mesma metodologia, que $75,75 \%$ das raízes se encontram nos primeiros $45,00 \mathrm{~cm}$ de profundidade.

De acordo Warrick \& Nielsen (1980) citado por Silva \& Chaves (2001) pode-se

\section{MATERIAL E MÉTODOS}

A pesquisa foi instalada em uma área experimental (latitude 6 $6^{\circ} 54^{\prime} 59,88^{\prime}$ 'S, longitude $35^{\circ} 09^{\prime} 17,86^{\prime \prime} \mathrm{O}$ e altitude de classificar a variabilidade das variáveis estudadas em função do CV em três classes: baixa $\quad(\mathrm{CV}<12,00 \%), \quad$ média $(12,00<\mathrm{CV}<62,00 \%)$ e alta $(\mathrm{CV}>62,00 \%)$. Silva \& Chaves (2001) recomendam o teste KS como forma de avaliar a distribuição de frequiência dos dados e verificar a sua aderência à distribuição normal.

Para Costa Neto (1997), o teste KS consiste no cálculo das diferenças entre a probabilidade das variáveis normais reduzidas e a probabilidade acumulada dos dados experimentais. Segundo o mesmo autor, se o valor calculado em módulo for menor que o tabelado a distribuição experimental é aceita como aderida à distribuição normal.

De acordo com Spiegel (1985), pela estatística descritiva, quando o coeficiente de curtose for igual a 3,00 fica caracterizada a distribuição normal dos dados; quando os resultados do teste KS forem igual a 5,00\% de probabilidade e os valores das medidas de tendência central, média e mediana, forem semelhantes, indicam, também, que os dados se distribuem de forma normal e, sendo assim, a média aritmética dos mesmos pode ser usada para inferir sobre a variabilidade das amostras.

O trabalho, desenvolvido em cana-de-açúcar sob diferentes lâminas de irrigação, tem como objetivo estudar o padrão de comportamento do crescimento radicular da variedade RB 92579 desenvolvendo um modelo geoespacial para os diferentes tratamentos de irrigação e correlacionar tal padrão de crescimento e de desenvolvimento com a produtividade final da cultura.

121,00 m) da Fazenda Capim II (Figura 1), localizada no Município de Capim/PB. 


\section{CRESCIMENTO DO SISTEMA RADICULAR DE CANA-DE-AÇÚCAR SUBMETIDA A LÂMINAS DE IRRIGAÇÃO: MODELO GEOESPACIAL E INFLUÊNCIAS NA PRODUTIVIDADE DA CULTURA}

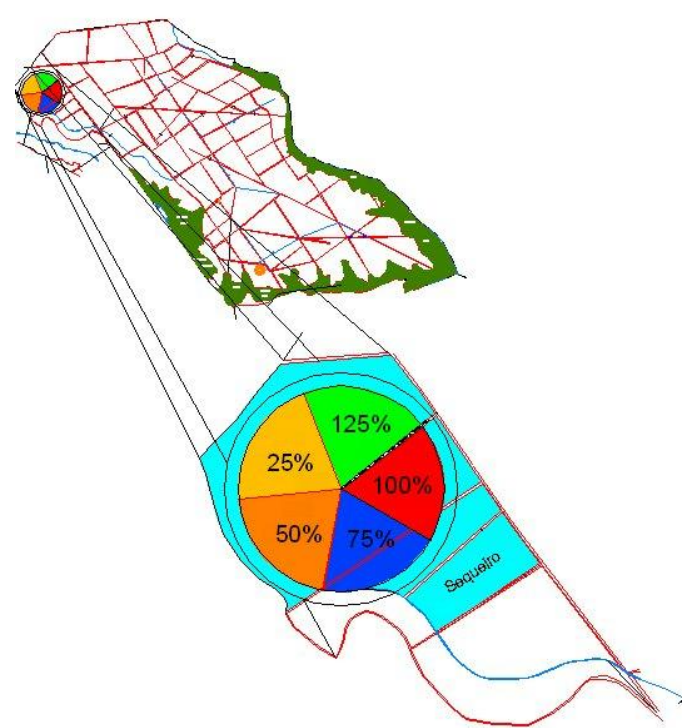

Figura 1. Croqui de localização do experimento na Fazenda Capim II e croqui da área experimental

A área possui relevo plano e solos com predominância dos Argissolos distróficos. A área total considerada como unidade experimental foi de 27,00 ha. A irrigação se deu via pivô central fixo da marca Valley ${ }^{\circledR} \mathrm{O}$ comprimento do centro do pivô até a última torre é de $257,4 \mathrm{~m}$, com um raio irrigado de $290 \mathrm{~m}$, em uma velocidade máxima de 127 $\mathrm{m} \mathrm{h}^{-1}$ quando o percentímetro está regulado em $100 \%$. O conjunto motor-bomba é formado por um motor $\mathrm{WEG}^{\circledR}$ de $75 \mathrm{cv}$ e uma bomba KSB com vazão de $172,8 \mathrm{~m}^{3} \mathrm{~h}^{-1}$. As lâminas de irrigação foram determinadas a partir da evapotranspiração (ETo); para a estimativa da ETc. A determinação da ETo foi feita utilizando-se o modelo de Penmann \& Monteith (Allen et al., 1998), sendo essa equação a mais apropriada para a região (Mendonça, 2008). Os dados, oriundos da Plataforma de Coleta de Dados (PCD) Capim do INPE, foram base para o cálculo da ETo conforme modelo acima citado e abaixo descrito.
ETo $=\frac{0,408 \Delta\left(R_{n}-G\right)+\gamma \frac{900}{T_{\text {med }}+273} u_{2}\left(e_{s}-e_{a}\right)}{\Delta+\gamma\left(1+0,34 u_{2}\right)}$

Em que: $\mathrm{R}_{\mathrm{n}} \mathrm{o}$ saldo de radiação total diário (MJ m ${ }^{-2} \mathrm{~d}^{-1}$ ); $\mathrm{G}$ a densidade de fluxo de calor no solo $\left(\mathrm{MJ} \mathrm{m}^{-2} \mathrm{~d}^{-1}\right) ; \mathrm{T}_{\text {med }}$ a temperatura média diária do ar a $2 \mathrm{~m}$ de altura $\left({ }^{\circ} \mathrm{C}\right) ; \mathrm{U}_{2}$ a velocidade do vento média diária a $2 \mathrm{~m}$ de altura $\left(\mathrm{m} \mathrm{s}^{-1}\right)$; $\mathrm{e}_{\mathrm{s}}$ a pressão de saturação do vapor média diária $(\mathrm{kPa})$; $\mathrm{e}_{\mathrm{a}}$ a pressão parcial de vapor média diária $(\mathrm{kPa}) ; \Delta$ a declividade da curva de pressão de saturação de vapor; $\square$ a constante psicrométrica igual a $0,0633 \mathrm{kPa}{ }^{\circ} \mathrm{C}^{-1}$.

Para o início do experimento a primeira irrigação foi realizada para elevar a umidade do solo à capacidade de campo em todos os setores. Para o balanço hídrico, a umidade do solo foi sempre estimada, abatendo-se as chuvas efetivas ( $74 \%$ da precipitação) da evapotranspiração da cultura (ETc) acumulada no turno de irrigação, levando-se em consideração a quantidade atual da água no solo no momento da irrigação (Tabela 1). 


\section{CRESCIMENTO DO SISTEMA RADICULAR DE CANA-DE-AÇÚCAR SUBMETIDA A LÂMINAS DE IRRIGAÇÃO: MODELO GEOESPACIAL E INFLUÊNCIAS NA PRODUTIVIDADE DA CULTURA}

Tabela 1. Lâmina de água de irrigação $(\mathrm{mm})$, precipitação $(\mathrm{mm})$, chuvas aproveitáveis $(\mathrm{mm})$ e lâmina total aplicada à cultura (mm).

\begin{tabular}{ccccc}
\hline Tratamento & Precipitação & $\begin{array}{c}\text { chuvas } \\
\text { aproveitáveis }\end{array}$ & $\begin{array}{c}\text { Lâmina de } \\
\text { irrigação }\end{array}$ & Água total \\
\hline Sequeiro & & 943,50 & 0 & 943,50 \\
$50 \%$ da ETc & & 876,42 & 191,05 & $1.067,47$ \\
$75 \%$ da ETc & $1.502,03$ & 705,86 & 379,31 & $1.085,17$ \\
$100 \%$ da ETc & & 567,24 & 680,08 & $1.247,32$ \\
$125 \%$ da ETc & & 394,67 & 913,95 & $1.308,62$ \\
\hline
\end{tabular}

No cálculo da evapotranspiração da cultura (ETc) foi utilizado um coeficiente de cultura (Kc) de acordo Doorembos \& Kassam (1994) para os diferentes estádios de desenvolvimento, como forma de determinar a evapotranspiração da cultura para cada estádio.

Foram coletadas, no plano tridimensional $(\mathrm{x}, \mathrm{y}, \mathrm{z})$ com três repetições; cada uma delas com um total de 216 amostras de solo com raízes. Para a amostragem foi utilizada um trado holandês para extração de raízes com base circular de diâmetro de 7,62 cm e altura de $15,00 \mathrm{~cm}$. As amostras, foram coletadas em um cubo de $1 \mathrm{~m}^{3}$ onde, ao centro estava a linha de cana.

$\mathrm{O}$ espaçamento entre amostras no plano $\mathbf{x y}$ foi de 20,00 x $20,00 \mathrm{~cm}$ e no plano $\mathbf{x z}$ e $\mathbf{y z}$ foi, em ambos, de 20,00 x 15,00 $\mathrm{cm}$ de profundidade (Figura 1). A separação entre o solo e as raízes se deu com o auxilio de uma peneira com malha de $2,00 \mathrm{~mm}$; as raízes coletadas foram secas em estufa de circulação fechada com temperatura regulada em $65,00{ }^{\circ} \mathrm{C}$ por $48: 00$ h. Após a secagem foi definido que se trabalharia com os dados convertidos para mg de raízes por ponto coletado para realização da análise geoespacial.

A técnica empregada neste trabalho para avaliar a dependência espacial dos dados amostrados foi a da geoestatística com os estudos de estacionaridade dos dados através do semivariograma, que é por definição:

$\gamma(h)=\frac{1}{2 N(h)} \sum_{i=1}^{N(h)}\left[Z\left(x_{i}\right)-Z\left(x_{i}+h\right)\right]^{2}$

Em que: $\mathrm{N}(\mathrm{h})$ é o número de pares de valores medidos $\mathrm{Z}\left(\mathrm{x}_{\mathrm{i}}\right), \mathrm{Z}\left(\mathrm{x}_{\mathrm{i}}+\mathrm{h}\right)$, separados por um vetor $h$. e os seus coeficientes teóricos foram definidos: o efeito pepita (Co); o patamar (C) e o alcance de dependência espacial (Ao) (Vieira, 2000).

Cambardella et al. (1994) citam que para o estudo de dependência espacial a interpretação da relação entre efeito pepita e o patamar se faz necessário e deve ser vista da seguinte forma: $\mathrm{Co} \leq 25,00 \%$ do $\mathrm{C}$ forte dependência espacial, $25,00 \%<\mathrm{Co}<75,00 \%$ do $\mathrm{C}$ dependência espacial moderada e Co $>75,00 \%$ do $\mathrm{C}$ dependência espacial fraca. 


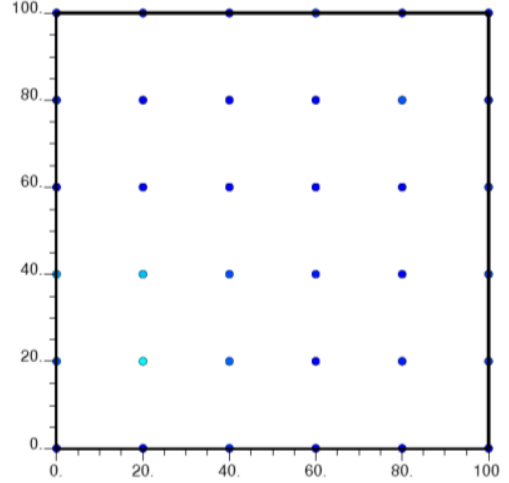

A

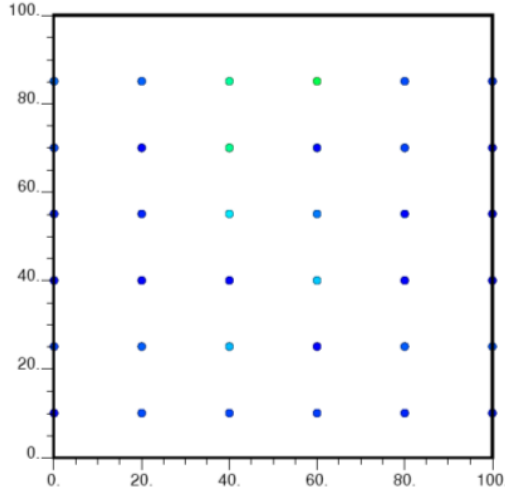

B

Figura 2. "Post script" da coleta de solo, em A o espaçamento 20 x $20 \mathrm{~cm}$ e em B o espaçamento 20 x $15 \mathrm{~cm}$.

Sistema radicular

Com a análise estatística verificou-se que os dados não se aderem a curva normal e que a análise geoespacial (Tabela 2) dos dados nas três condições estudadas é uma tecnologia altamente viável no estudo do sistema

\section{RESULTADOS E DISCUSSÃO}

radicular em cana-de-açúcar, inclusive com dependência espacial de moderada a forte, o que permite assim a estimativa de variáveis como massa total volume de solo explorado e densidade (Tabela 3) e visualização em 3D (Figura 2).

Tabela 2. Estatística descritiva e análise geoestatística para o comportamento do crescimento do sistema radicular de cana-de-açúcar, variedade RB 92579 nos seis tratamentos.

\begin{tabular}{|c|c|c|c|c|c|c|c|c|c|}
\hline \multirow{2}{*}{$\begin{array}{l}\text { Água total } \\
\text { (mm) }\end{array}$} & \multirow{2}{*}{$\mathrm{CV}$} & \multirow{2}{*}{ Média } & \multirow{2}{*}{ Moda } & \multirow{2}{*}{ Mediana } & \multicolumn{2}{|c|}{ Valores } & \multicolumn{3}{|c|}{ Teste KS * } \\
\hline & & & & & Mínimo & Máximo & Dmax & $\mathrm{Dt}^{* *}$ & $\begin{array}{l}\text { Dst. } \\
\text { Norm }\end{array}$ \\
\hline 943,50 & 1,41 & 76,21 & 0 & 42,52 & 0 & 652,73 & 0,241 & 0,1177 & Não \\
\hline $1.067,47$ & 1,25 & 127,65 & 0 & 90,03 & 0 & 1018,53 & 0,213 & 0,1177 & Não \\
\hline $1.085,16$ & 1,42 & 103,87 & 0 & 58,62 & 0 & 1195,67 & 0,242 & 0,1177 & Não \\
\hline $1.247,32$ & 1,60 & 60,88 & 0 & 29,96 & 0 & 635,90 & 0,267 & 0,1177 & Não \\
\hline \multirow[t]{3}{*}{$1.308,61$} & 2,02 & 42,74 & 0 & 7,86 & 0 & 632,27 & 0,312 & 0,1177 & Não \\
\hline & \multirow{2}{*}{\multicolumn{2}{|c|}{$\mathrm{C}_{\mathrm{o}}$}} & \multirow{2}{*}{$\mathrm{C}$} & \multirow{2}{*}{\multicolumn{2}{|c|}{ Alcance }} & \multicolumn{2}{|c|}{$\left[\mathrm{C}_{\mathrm{o}} / \mathrm{C}\right] \times 100$} & \multirow{2}{*}{\multicolumn{2}{|c|}{ Modelo }} \\
\hline & & & & & & Valor & Dep. espacial & & \\
\hline 943,50 & \multicolumn{2}{|c|}{0,15} & 0,805 & \multicolumn{2}{|r|}{25} & 18,63 & Forte & \multicolumn{2}{|c|}{ Esférico } \\
\hline $1.067,47$ & \multicolumn{2}{|c|}{0,10} & 0,530 & \multicolumn{2}{|r|}{23} & 18,86 & Forte & \multicolumn{2}{|c|}{ Esférico } \\
\hline $1.085,16$ & \multicolumn{2}{|c|}{0,18} & 0,620 & \multicolumn{2}{|r|}{28} & 29,03 & Moderada & \multicolumn{2}{|c|}{ Esférico } \\
\hline $1.247,32$ & \multicolumn{2}{|c|}{0,25} & 0,765 & \multicolumn{2}{|r|}{22} & 32,67 & Moderada & \multicolumn{2}{|c|}{ Esférico } \\
\hline $1.308,61$ & \multicolumn{2}{|r|}{0,25} & 0,769 & \multicolumn{2}{|r|}{28} & 32,50 & Moderada & \multicolumn{2}{|c|}{ Esférico } \\
\hline
\end{tabular}

${ }^{1}$ Dados em mg considerando o volume de solo de $742,71 \mathrm{~cm}^{3}$, ${ }^{*} \mathrm{KS}$-teste de Kolmogorov-Smirnov te; $\mathrm{D}_{\max }-$ diferença absoluta entre a função de distribuição normal acumulada e a freqüência relativa observada acumulada e ajustada, $\mathrm{D}_{\mathrm{t}}$-valor crítico tabelado para uma amostra n e $\delta=0,5$ com significância de $5 \%(\alpha=0,05), \mathrm{D}_{\max }>\mathrm{Dt}$; distribuição não normal, $\mathrm{Co}$ - efeito pepita, $\mathrm{C}$ - patamar 


\section{CRESCIMENTO DO SISTEMA RADICULAR DE CANA-DE-AÇÚCAR SUBMETIDA A LÂMINAS DE IRRIGAÇÃO: MODELO GEOESPACIAL E INFLUÊNCIAS NA PRODUTIVIDADE DA \\ CULTURA}

Da Tabela 3, depreende-se que a cana sob condições de sequeiro desenvolveu 44,48\% da sua massa total de raiz nos primeiros $68,75 \mathrm{~cm}$ de profundidade explorando $11,06 \%$ do volume de solo estudado $\left(110.625 \mathrm{~cm}^{3}\right)$ caracterizando a zona de maior densidade, interpretadas como sendo as raízes de sustentação, enquanto a de baixa densidade se localizou ao longo de todo o perfil estudado, concentrado 55,51\% da massa total do sistema radicular em $67,12 \%$ do volume de solo estudado $\left(671.234,38 \mathrm{~cm}^{3}\right)$. A cana de sequeiro deixou de explorar, por falta de raiz, um total de $21,81 \%$ do volume de solo estudado.

Tabela 3. Estimativas a partir da análise geoestatística para a Massa Total de Raízes estimada (MTRe), volume de solo explorado (V) Profundidade (P) e Densidade D (g de raízes $/ \mathrm{cm}^{3}$ de solo).

\begin{tabular}{|c|c|c|c|c|c|c|c|c|}
\hline $\begin{array}{l}\text { Trat. } \\
\text { (ETc) }\end{array}$ & $\begin{array}{l}\text { Água total } \\
\quad(\mathrm{mm})\end{array}$ & $\begin{array}{l}\text { MTRe } \\
(\mathrm{kg})\end{array}$ & Zona & $\mathrm{M}(\mathrm{kg})$ & $\mathrm{V}\left(\mathrm{cm}^{3}\right)$ & $\%$ & $\mathrm{P}(\mathrm{cm})$ & $\begin{array}{c}\mathrm{D} \\
\left(\mathrm{mg} / \mathrm{cm}^{3}\right)\end{array}$ \\
\hline \multirow{5}{*}{ Seq } & \multirow{5}{*}{943,50} & \multirow{5}{*}{4,56} & Rosada & 0,86 & $36.468,75$ & 19,04 & $0-33,75$ & 23,58 \\
\hline & & & Verde & 1,15 & $74.156,25$ & 25,44 & $0-68,75$ & 15,51 \\
\hline & & & Vermelha & 1,13 & $122.906,25$ & 24,97 & $0-98,75$ & 9,19 \\
\hline & & & Azul & 1,39 & $548.328,13$ & 30,54 & $0-98,75$ & 2,53 \\
\hline & & & Amarela & 0,00 & $218.140,62$ & 0,00 & $18,75-98,75$ & 0,00 \\
\hline \multirow{5}{*}{$50 \%$} & \multirow{5}{*}{$1.067,47$} & \multirow{5}{*}{7,84} & Rosada & 0,52 & $13.843,75$ & 6,63 & $0-33,75$ & 37,56 \\
\hline & & & Verde & 1,68 & $70.875,00$ & 21,49 & $0-46,25$ & 23,70 \\
\hline & & & Vermelha & 4,85 & $447.812,50$ & 61,89 & $0-68,75$ & 10,83 \\
\hline & & & Azul & 0,78 & $352.828,13$ & 9,97 & $0-98,75$ & 2,21 \\
\hline & & & Amarela & 0,00 & $114.640,63$ & 0,00 & $11,25-98,75$ & 0,00 \\
\hline \multirow{5}{*}{$75 \%$} & \multirow{5}{*}{$1.085,16$} & \multirow{5}{*}{6,41} & Rosada & 0,30 & $9.500,00$ & 4,70 & $6,25-38,75$ & 31,58 \\
\hline & & & Verde & 2,05 & $89.046,88$ & 32,05 & $0-43,75$ & 23,02 \\
\hline & & & Vermelha & 3,03 & $289.046,88$ & 47,40 & $0-68,75$ & 10,48 \\
\hline & & & Azul & 1,02 & $334.750,00$ & 15,85 & $0-98,75$ & 3,05 \\
\hline & & & Amarela & 0,00 & $277.656,25$ & 0,00 & $13,25-98,25$ & 0,00 \\
\hline \multirow{5}{*}{$100 \%$} & \multirow{5}{*}{$1.247,32$} & \multirow{5}{*}{2,86} & Rosada & 0,03 & $13.575,00$ & 1,07 & $0-36,25$ & 2,21 \\
\hline & & & Verde & 0,22 & $14.593,75$ & 7,82 & $0-38,75$ & 15,07 \\
\hline & & & Vermelha & 1,04 & $117.703,13$ & 36,39 & $0-53,75$ & 8,84 \\
\hline & & & Azul & 1,56 & $555.221,87$ & 54,7 & $0-98,75$ & 2,81 \\
\hline & & & Amarela & 0,00 & $298.906,25$ & 0,00 & $0-98,75$ & 0,00 \\
\hline \multirow{5}{*}{$125 \%$} & \multirow{5}{*}{$1.308,61$} & \multirow{5}{*}{2,20} & Rosada & 0,32 & $14.953,13$ & 14,70 & $0-38,75$ & 21,40 \\
\hline & & & Verde & 0,38 & $25.187,50$ & 17,39 & $0-43,75$ & 15,09 \\
\hline & & & Vermelha & 0,64 & $74.048,13$ & 29,30 & $0-51,25$ & 8,64 \\
\hline & & & Azul & 0,85 & $387.936,24$ & 38,60 & $0-98,75$ & 2,19 \\
\hline & & & Amarela & 0,00 & $497.875,00$ & 0,00 & $0-98,75$ & 0,00 \\
\hline
\end{tabular}




\section{CRESCIMENTO DO SISTEMA RADICULAR DE CANA-DE-AÇÚCAR SUBMETIDA A LÂMINAS DE IRRIGAÇÃO: MODELO GEOESPACIAL E INFLUÊNCIAS NA PRODUTIVIDADE DA CULTURA}

A cana irrigada com $50 \%$ da ETc mostrou um padrão de comportamento bem definido; sendo que os primeiros $46,25 \mathrm{~cm}$ de profundidade foram ocupados por $28,12 \%$ da massa total do sistema radicular que são de alta concentração, interpretadas como sendo raízes de sustentação e exploraram um volume de solo equivalente a $8,47 \%$ do total estudado; $71,86 \%$ do sistema radicular dessa cana estão na zona de baixa concentração radicular (interpretados como sendo zona de pelos absorventes) e se distribuem ao longo de todo o perfil estudado, explorando $77,06 \%$ do volume de solo estudado. Para esse tratamento, desde os $11,25 \mathrm{~cm}$ até os $98,75 \mathrm{~cm}$ de profundidade encontrou-se pontos sem sistema radicular que totalizaram $11,46 \%$ do volume total de solo, o que pode ser visualizado na Figura 2.

Observou-se que a cana irrigada com $75 \%$ da ETc desenvolveu 36,75\% do seu sistema

Quando a cultura foi irrigada com $125 \%$ da ETc; 32,09\% da massa de raízes foram encontradas nos primeiros 43,75 $\mathrm{cm}$ de profundidade e destes $14,70 \%$ sendo raízes de sustentação (zona de alta densidade). $67,90 \%$ do sistema radicular permaneceram na zona de baixa concentração radicular (interpretados como sendo zona de pelos absorventes) e se distribuíram ao longo de todo o perfil estudado, desde a superfície até os $98,75 \mathrm{~cm}$ de profundidade. Observou-se que quando irrigada com $125 \%$ da ETc, a cana diminuiu a emissão de raízes ao longo do perfil estudado; verificou-se que, nesse caso $49,78 \%$ do solo não foi explorado pelas raízes da cana.

Dos resultados encontrados nesta pesquisa, depreende-se que na medida em que se aumenta a lâmina de água incidida na cultura, superficializam-se as zonas de alta densidade e em contrapartida se aumenta a emissão de raízes finas formando zonas de baixa densidade. Tais resultados corroboram com o que diz Morris e Tai (2004). Para Otto et al. (2009) a utilização de um trado para coleta de raízes foi viável na obtenção nos primeiros $43,75 \mathrm{~cm}$ de profundidade. Nela, a zona de maior densidade, interpretadas como sendo as raízes de sustentação, está concentrada na faixa de 6,25 a $38,75 \mathrm{~cm}$ enquanto a de baixa densidade se localizou ao longo de todo o perfil estudado concentrado $63,27 \%$ do sistema radicular. Nesse tratamento, 27,76\% do volume de solo estudado foi inexplorado pelas raízes da cana, ou seja não houve presença das mesmas.

Para a cana irrigada com $100 \%$ da demanda observou-se que $8,87 \%$ das raízes situaramse nos primeiros $38,75 \mathrm{~cm}$ de profundidade e uma zona cuja densidade esteve entre 2,21 a $15,07 \mathrm{mg} \mathrm{cm}^{-3}$ de solo. Da Tabela 3 depreende-se que $54,70 \%$ da massa total de raízes possuem densidade de $2,81 \mathrm{mg} \mathrm{cm}^{-3}$ de solo. A cana quando irrigada com $100 \%$ da ETc deixou 29,89\% do volume do solo estudado sem exploração, ou seja sem raízes.

dos dados como afirmam, também, Farias et al. (2008).

Produtividade e correlações com a Massa Total de Raízes estimada

Na Tabela 4 podem ser observados os efeitos significativos para a irrigação; dela depreende-se que muito embora a safra ora analisada, tenha sido de regular pluviometria os efeitos da irrigação foram altamente significativos do ponto de vista da produtividade física.

A diferença entre a cana irrigada com $125 \%$ da ETc para a cana cultivada em regime de Sequeiro foi de $28,69 \%$, entretanto para a cana irrigada com 50 e $75 \%$ da ETc a diferença é de apenas $0,91 \%$ e entre a cana irrigada com 100 e $125 \%$ da ETc a diferença é de $0,57 \%$.

Entre a cana irrigada com $100 \%$ da ETc e aquela irrigada com $75 \%$ da ETc a diferença foi de 6,29\%. Na Tabela 4, observa-se que foi significativo (com $5 \%$ de probabilidade) o modelo de regressão com o qual se estimou a massa de raízes em função da produtividade alcançada. E notório que a 


\section{CRESCIMENTO DO SISTEMA RADICULAR DE CANA-DE-AÇÚCAR SUBMETIDA A LÂMINAS DE IRRIGAÇÃO: MODELO GEOESPACIAL E INFLUÊNCIAS NA PRODUTIVIDADE DA CULTURA}

presença de uma grande massa de raízes não é sinônimo de maiores produtividades. Com o uso do modelo constante na Figura 3, observa-se que o ponto de máximo desenvolvimento radicular, ou seja, máxima produção de raízes $\left(8,31 \mathrm{~kg} \mathrm{~m}^{-3}\right)$ se dá quando a produtividade atinge $130,12 \mathrm{t} \mathrm{ha}^{-1}$. Entretanto, quando a produtividade aumenta para patamares em torno de 145,62 $\mathrm{t} \mathrm{ha}^{-1}$ a massa radicular decresce para $2,25 \mathrm{~kg} \mathrm{~m}^{-3}$.
Tais resultados encontrados nessa pesquisa encontram fundamentação com o trabalho desenvolvido por Morris e Tai (2004). Os autores concluíram que os melhores rendimentos estão sempre relacionados à menor concentração de raízes curtas e de diâmetro maior na superfície e maior concentração de raízes longas e mais finas nos horizontes mais profundos do solo.

Tabela 4. Resumo da análise de variância para os índices de produção e análise de regressão entre a produtividade a massa total de raízes estimada a variedade RB 92579 submetida a lâminas de irrigação

\begin{tabular}{|c|c|c|c|c|c|c|c|c|c|c|}
\hline \multirow[b]{3}{*}{ Var } & \multicolumn{10}{|c|}{ Anova para produtividade $\left(\mathrm{t} \mathrm{ha}^{-1}\right)$} \\
\hline & & ndices est & ísticos a & nalisados & & & & Médias & & \\
\hline & $\begin{array}{c}\text { Blc/lâ } \\
\text { m }\end{array}$ & Lâmina & QMR & $\mathrm{CV}(\%)$ & Dms & Seq & $50 \%$ & $75 \%$ & $100 \%$ & $125 \%$ \\
\hline Prod & $\underset{\mathrm{ns}}{425,66}$ & $3087,57^{*}$ & $\begin{array}{c}234,3 \\
0\end{array}$ & 11,63 & $\begin{array}{c}16,5 \\
9\end{array}$ & $\begin{array}{c}113,1 \\
5\end{array}$ & $\begin{array}{c}134,9 \\
8\end{array}$ & 136,21 & $\begin{array}{c}144,7 \\
9\end{array}$ & $\begin{array}{c}145,6 \\
2\end{array}$ \\
\hline
\end{tabular}

Análise de regressão para Massa Total de Raízes estimada (MTRe), em função da produtividade

\begin{tabular}{ccccccccc}
\hline & Coeficiente & DP & T & Coef. beta & $\begin{array}{c}\text { Prob } \\
(\text { coe })\end{array}$ & $\mathrm{R}^{2}$ & F (eq) & $\begin{array}{c}\text { Prob } \\
(\mathrm{eq})\end{array}$ \\
\hline Constante & $-295,146$ & & & & & & & \\
Prod & 4,76301 & $\begin{array}{c}0,579 \\
9\end{array}$ & 8,2134 & 26,3804 & $0,0073^{* *}$ & 94,9 & 38,60 & 0,0253 \\
Prod $^{2}$ & $-0,018684$ & $\begin{array}{c}0,002 \\
2\end{array}$ & $-8,3175$ & $-2,67148$ & $0,0071^{* *}$ & 5 & & \\
\hline
\end{tabular}

"** significativo com 1\%; "significativo com 5\%; ${ }^{\text {ns }}$ não significativo pelo teste F; QMR - quadrado médio do resíduo; $\mathrm{CV}(\%)$ - coeficiente de variação; QMDR - quadrado médio devido a regressão; $\mathrm{F}$ valor de F calculado; Prob, F - significância do modelo; TCH- toneladas de cana por hectares; DP Desvio padrão.

Para a cana irrigada com $100 \%$ da ETc $91,09 \%$ do seu sistema radicular foi de baixa densidade $\left(\mathrm{mg} / \mathrm{cm}^{3}\right)$, constituindo-se de material mais fino e leve (radicelas) que são mais eficientes na absorção de nutrientes. Quando a cana é irrigada com $125 \%$ da ETc esse total é de $69,70 \%$. Nesse sentido, na cana em regime hídrico de sequeiro 90,01\% do seu sistema radicular é considerado como de média a alta densidade (raízes mais grossas e pesadas).

\section{CONCLUSÕES}

A análise geoestatística para a definição do modelo geoespacial do crescimento radicular da cana-de-açúcar sob condições de sequeiro e irrigada com e 50, 75, 100 e $125 \%$ da ETc pode ser utilizada com confiabilidade e acurácia na modelagem de seu desenvolvimento e de sua distribuição no espaço.

A irrigação promoveu elevada produtividade, superficialização da zona de alta densidade radicular $\mathrm{e}$ favoreceu $\mathrm{o}$ aumento de raízes em zonas de baixa densidade. 


\section{CRESCIMENTO DO SISTEMA RADICULAR DE CANA-DE-AÇÚCAR SUBMETIDA A LÂMINAS DE IRRIGAÇÃO: MODELO GEOESPACIAL E INFLUÊNCIAS NA PRODUTIVIDADE DA CULTURA}

Maior massa de raízes não é uma condição "sine qua non" para obtenção de elevada produtividade da cana-de-açúcar, variedade RB 92 579, haja vista o fato de que a variedade estudada diminui a produção de sua massa radicular quando em condições de maiores produtividade.

\section{AGRADECIMENTO}

À Destilaria Miriri S.A. pelo incentivo, fomento e dedicação à pesquisa com cana-de-açúcar.

\section{REFERENCIAS BIBLIOGRÁFICAS}

ALLEN, R.G.; PEREIRA, L.S.; RAES, D.; SMITH, M. Crop evapotranspiration Guidelines for predicting crop water requeriments, Rome: FAO, 300p. (FAO. Irrigation and Drainage Paper, 56), 1998.

COSTA NETO, P.L.O. Estatística. 15 ed. São Paulo: Edgard Blucher, 468p. 1997.

CAMBARDELLA, C. A; MOORMAN, T. B; NOVACK, J. M; PARKIN, T.B;

DOORENBOS, J.; KASSAM, A. H. Efeito da água no rendimento das culturas. Campina Grande: UFPB, 306 p. (FAO. Estudos de Irrigação e Drenagem, 33), 1994.

FARIAS, C.H. de A.; OLIVEIRA, K.S. de; ANTUNES, V.L.; SILVA, P.C.M. da. Variabilidade química do solo dos tabuleiros costeiros paraibanos I: fósforo, cálcio e alumínio. XXXIII Congresso Brasileiro de Engenharia Agrícola. CD ROM, São Pedro, SP, 4p. 2004.

FARIAS, C.H. de A., OLIVEIRA G.S. de. Alumínio: variabilidade em tabuleiro costeiro paraibano e sua influência no cultivo da cana-de-açúcar. XXXVI Congresso Brasileiro de Engenharia Agrícola. CD ROM. 4p, Bonito/MS. 2007.

FARIAS, C. H. de A.; FERNANDES, P. D.; AZEVEDO, H. M. \& DANTAS NETO J. Índices de crescimento da cana-de-açúcar irrigada e de sequeiro no Estado da Paraíba. Revista Brasileira de Engenharia Agrícola e Ambiental, v.12, n.4, p.356-362, 2008.

MENDONÇA, E.A. Estimativa da evapotranspiração de referência no município de Capim-PB. Dissertação
(Mestrado em Meteorologia). Universidade Federal de Campina Grande, Campina Grande, 114p., 2008.

MORRIS, D.R. \& TAI, P.Y.P.Water table effects on sugarcane root and shoot development. Journal American Society Sugar Cane Technologists, Vol. 24, 2004

OTTO, R.; TRIVELIN, P. C.O.; FRANCO, H. C. J.; FARONI, C. E. \&VITTI, A.C. Root system distribution of sugarcane as related to nitrogen fertilization, evaluated by two methods: monolith and probes. Revista Brasileira de Ciência do Solo. V.33, p.601611, 2009.

SPIEGEL, M. R. Estatística. 2.ed. São Paulo: McGraw Hill, 1985, 454p.

SILVA, P.C.M. da \& CHAVES, L.H.G. Avaliação e variabilidade espacial de Fósforo, Potássio e matéria orgânica em alissolos. Revista Brasileira de Engenharia Agrícola e Ambiental. V.5, n.3, p.431-436, 2001.

ÚNICA - União da Agroindústria Canavieira do Estado de São Paulo. Disponível em: http://www.portalunica.com.br/portalunica/? $\underline{\text { Secao=UNICA\%20em\%20ação\&SubSecao }}$ =cana-de-açúcar. Acesso em: 01/01/2010.

VIEIRA, S.R. Geoestatística em estudos de variabilidade do solo. In: tópicos em ciência do solo, volume I, SBCS, Viçosa-MG, p.252,2000 . 


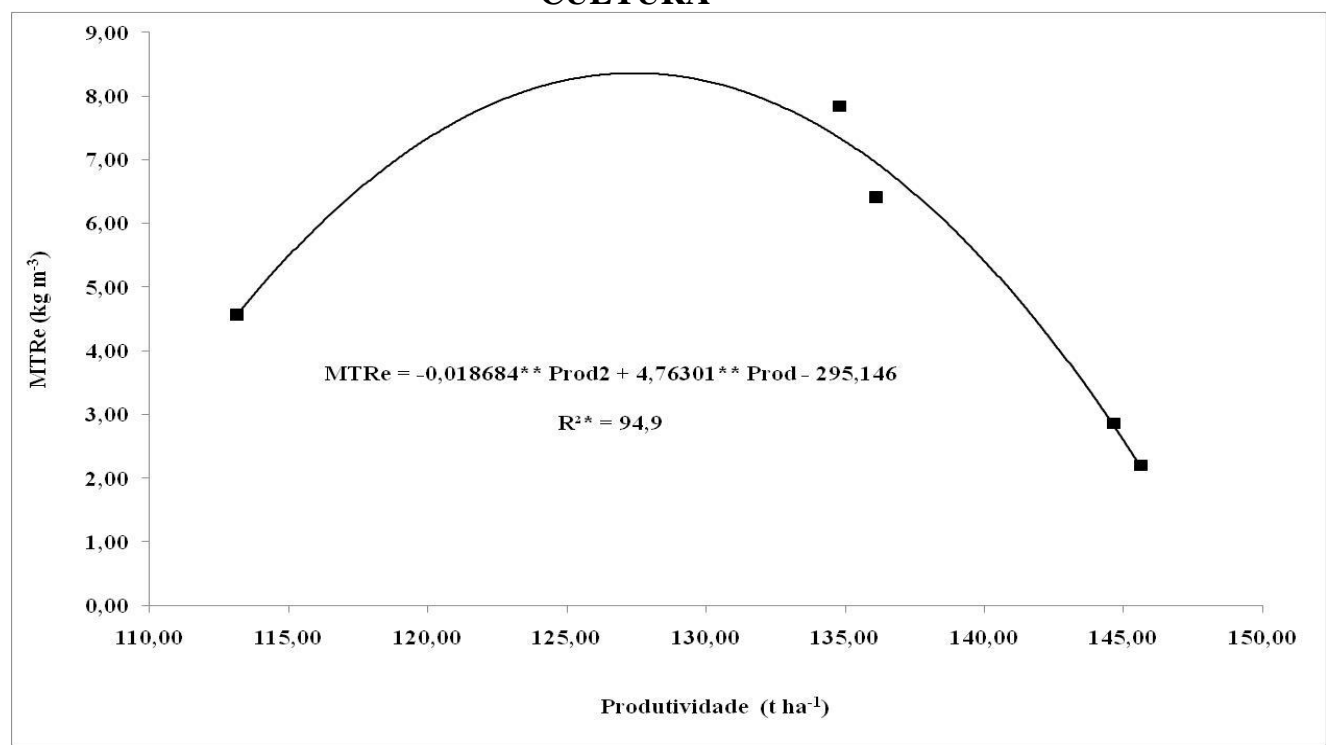

Figura 3. Modelo de regressão para a Massa Total de Raízes estimadas em função da produtividade alcançada sob lâminas de água total (mm).

\begin{tabular}{|c|c|c|c|}
\hline & Visualização em 3D & Histogramas & Semivariogramas \\
\hline 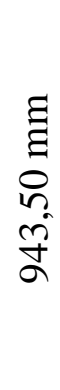 & & 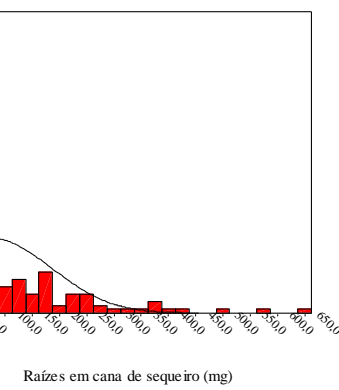 & 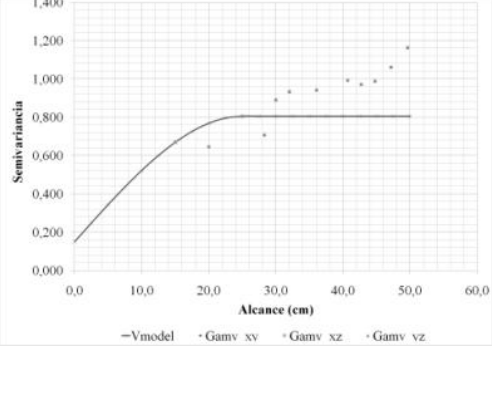 \\
\hline 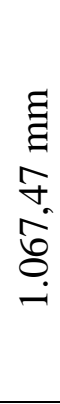 & & 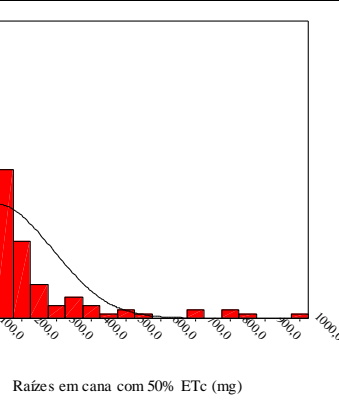 & 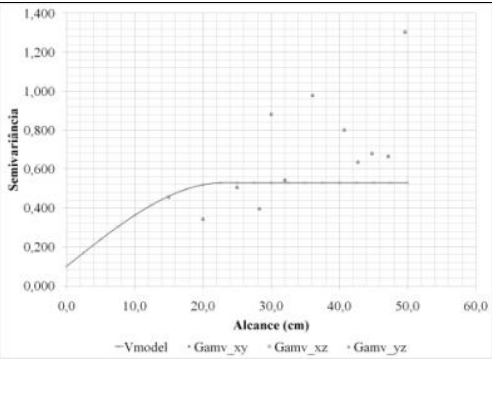 \\
\hline $\begin{array}{l}\Xi \\
\Xi \\
0 \\
\ddots \\
0 \\
0 \\
0\end{array}$ & & Rárese em cana com $75 \%$ da ETc (mg) & 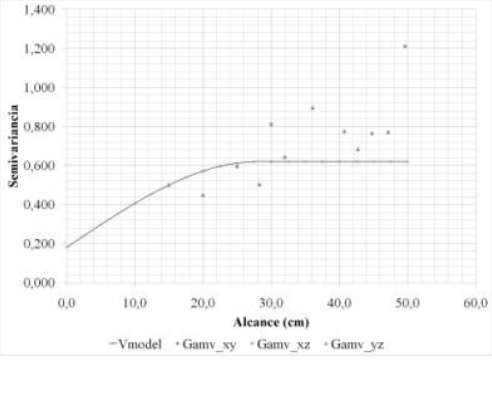 \\
\hline
\end{tabular}


CRESCIMENTO DO SISTEMA RADICULAR DE CANA-DE-AÇÚCAR SUBMETIDA A LÂMINAS

DE IRRIGAÇÃO: MODELO GEOESPACIAL E INFLUÊNCIAS NA PRODUTIVIDADE DA

\section{CULTURA}

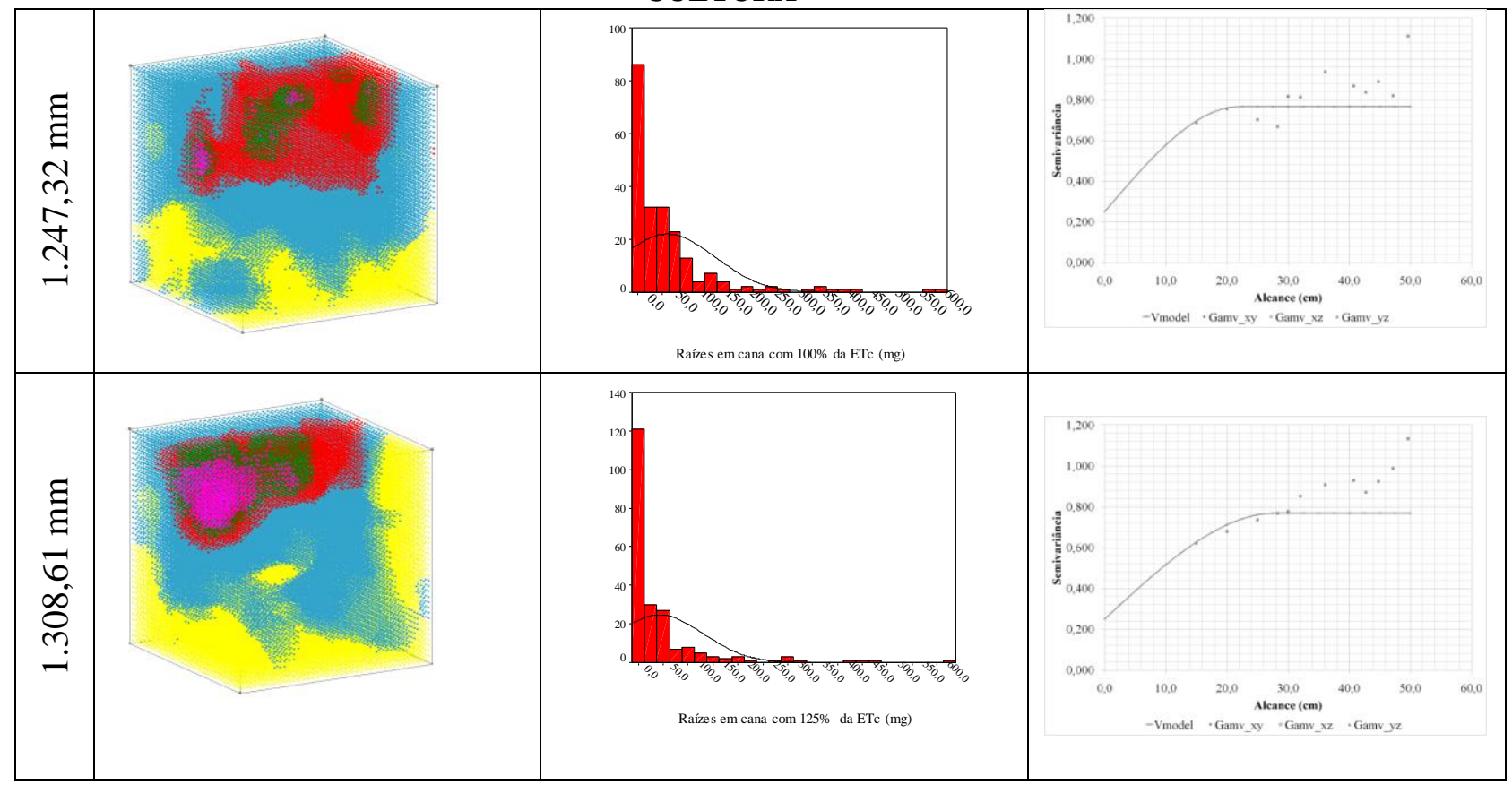

Figura 1. Visualização em 3D (x,y,z,c), estudo de freqüência dos dados e semivariogramas massa de raízes na cana de sequeiro e irrigada com 25, 50, 75100 e $125 \%$ da ETc, considerando a lâmina de água total incidida sobre a variedade RB 92579. 